\title{
Message from the PRISMO Workshop Organizers
}

These proceedings contain the papers selected for presentation at the first edition of the IEEE Workshop on Privacy and Security of Moving Objects (PRISMO'13) which is being held in conjunction with the IEEE MDM'13 conference.

Movement data constitutes valuable information. Data about user and object movement is a very rich source of information with social, economic and environmental value, and it is nowadays available in such a scale, that it is considered a characteristic case of big data. The information richness that makes movement data so valuable often poses a threat to the privacy and security of users and companies, who are behind the digital location and trajectory data. Movement data can reveal the exact location of a user in real time to third parties, which is a violation of user privacy by per se. But it is not only the user location that is revealed by location traces; studying the movement patterns of a user can reveal sensitive information as the location of her home, where she works, religious preferences and even indicate health problems. Often, context information can help to reveal the details of users behavior. Therefore protecting context data is mandatory to forestall the leak of sensitive behavior information.

The purpose of PRISMO is to encourage principled research that will lead to the advancement of the science of privacy and security on spatio-temporal data and in the study of the relationship between data protection and context. We hope that these proceedings will serve as a valuable reference for researchers and practitioners to motivate further research and development in mobility data security and privacy. This year's program is centered on privacy in LBS, geolocation services, geo-social networks and trajectory data publishing.

We should also mention that PRISMO'13 is a joint effort and we would like to especially thank the authors for providing the content of the program and the program committee for the evaluation process. We should also thank the organization committee of MDM'13 for the help with the workshop planning. We hope that these proceedings will inspire new research ideas, and you will enjoy reading them.

Maria Luisa Damiani, University of Milan, Italy

Bradley Malin, Vanderbilt University, USA

Anna Monreale, KDD Laboratory, University of Pisa, Italy

Yucel Saygin, Sabanci University, Istanbul, Turkey

Manolis Terrovitis, Research Center 'Athena', Athens, Greece 